Received: 29.11 .2018

Revised: 08.03.2019

Accepted: 22.03 .2019

DOI: $10.17804 / 2410-9908.2019 .2 .028-036$

\title{
ANALYSIS OF TWO METHODS FOR CALCULATING THE ULTIMATE STRESSES OF CREEP AND CREEP RUPTURE PROCESSES
}

\author{
V. V. Nazarov \\ Institute of Mechanics Lomonosov Moscow State University \\ Michurinsky prospect, 1, Moscow, 119192, Russian Federation \\ (iD https://orcid.org/0000-0002-9234-3646 inmec130@mail.ru \\ Corresponding author. E-mail: inmec130@mail.ru \\ Address for correspondence: Michurinsky prospect, 1, Moscow, 119192, Russian Federation
}

It is well known that structural elements and parts operating in a loaded state at high temperature can be subjected to irreversible deformation in time. For calculations of operating life (based on the corresponding mechanical models) may require the mechanical characteristics of the material, ones of which is the start creep stress and rupture stress (ultimate creep stresses) for a particular material at a given temperature. These mechanical characteristics can't be determined from the diagram of the mechanical state of the material (it is measured under uniaxial tensile tests of cylindrical specimens at a constant tensile stress over time). The complexity of such tests will require alternative methods of calculating the desired mechanical characteristics. In this paper, instead of special tests, it is proposed to calculate the desired mechanical characteristics from approximations of the secondary creep and creep-rupture processes. To this end, we considered two fractional power-law functions with four material parameters (S. A. Shesterikov and M. A. Yumasheva, 1984), two of which have the physical meaning of the start creep stress and rupture stress: approximation of the strain rate for secondary creep of the nominal stress (1), approximation of the rupture time of the nominal stress (2). The calculations used experimental data obtained for titanium alloy VT6 at $650{ }^{\circ} \mathrm{C}$ on cylindrical specimens (diameter is $5 \mathrm{~mm}$, base length is $25 \mathrm{~mm}$ ). The parameters of fractional-power approximations were calculated by the iterative method Generalized Reduced Gradient Method (Microsoft Excel) under the condition of the minimum total error of the discrepancy of the experimental data with respect to the approximating curve in the corresponding logarithmic axes. The analysis of approximation errors (1) and (2) showed that the error (1) is less than (2), and the ratio of the rupture stress to the start creep stress is greater. Based on the analysis of the results, it is concluded that it is better to use approximation (1) for calculating the ultimate creep stresses.

Keywords: ultimate creep stresses, start creep stress, rupture stress.

\section{References}

1. Naprienko S.A., Orlov M.R. Damage of single-crystal turbine blades of GTP. Electronic scientific journal "Proceedings of VIAM", 2016, vol. 38, no. 2, pp. 20-31. DOI: 10.18577/23076046-2016-0-2-3-3. (In Russian).

2. Ilyin S.I., Koryagin Yu.D., Lapina E.V. Creep of ultra-light magnesium alloys at low temperatures. Bulletin of the South Ural State University. Series Metallurgy, 2012, iss. 18, no. 15, pp.105-107. (In Russian).

3. Kachanov L.M. Osnovy mekhaniki razrusheniya [Fundamentals of Fracture Mechanics]. Moscow, Nauka Publ., 1974, 312 p. (In Russian). 
4. Shesterikov S.A., Yumasheva M.A. Specification of equation of state in creep theory. Izvestiya AN SSSR. Mekhanika Tverdogo Tela, 1984, no. 1, pp. 86-91. (In Russian).

5. Nazarov V.V., Lepeshkin A.R. A method of calculating creep limits. Diagnostics, Resource and Mechanics of materials and structures, 2017, iss. 1, pp. 36-42. Available at: http://dreamjournal.org/DREAM_Issue_1_2017_Nazarov_V.V._et_al._036_042.pdf

6. Lokoshchenko A.M., Nazarov V.V. Kinetic Approach to Studying the Creep Rupture of Metals under Biaxial Tension. Aviats.-Kosm. Tekh. Tekhnol., 2005, no. 10, pp. 73-78. (In Russian).

7. Lokoshchenk A.M., Agakhi K.A., Fomin L.V., Pure bending of a beam made of differently strength material under creeping. Vestn. Samarsk. Gos. Tekhn. Univ. Ser Fiz.-Mat. Nauki, 2012, no. 1 (26), pp. 66-73. (In Russian).

8. Fomin L.V. Description of creep rupture strength of tensile rod with rectangular and circular cross-section at high temperature air media. Vestn. Samar. Gos. Tekhn. Univ., Ser. Fiz.Mat. Nauki (J. Samara State Tech. Univ., Ser. Phys. Math. Sci.), 2013, iss. 3 (32), pp. 87-97. DOI: 10.14498/vsgtu1228. (In Russian).

9. Nazarov V.V. Short-Term Creep of Titanium Alloys VT5 and VT6 at High Temperature. Industrial Laboratory. Diagnostics of Materials, 2015, vol. 81, no. 6, pp. 57-60. (In Russian). Available at: https://www.zldm.ru/jour/article/view/88/89

10. Koval'kov V.K., Nazarov V.V., Novotnyi S.V. Procedure of high-temperature within complex stressed state. Zavod. Lab. Diagn. Mater., 2006, vol. 72, no. 4, pp. 42-44. (In Russian). 
Подана в журнал: 29.11.2018

УДК 620.172.2:620.162.3:539.376

DOI: $10.17804 / 2410-9908.2019 .2 .028-036$

\title{
АНАЛИЗ ДВУХ СПОСОБОВ ВЫЧИСЛЕНИЯ ПРЕДЕЛЬНЫХ НАПРЯЖЕНИЙ ПРОЦЕССОВ ПОЛЗУЧЕСТИ И ДЛИТЕЛЬНОЙ ПРОЧНОСТИ
}

\author{
В. В. Назаров
}

\author{
Научно-исследовательский институт механики \\ Федерального государственного бюджетного образовательного учреждения высшего образования \\ «Московский государственный университет им. М.В. Ломоносова» \\ Мичуринский проспект, 1, Москва, 119192, Российская Федерачия \\ https://orcid.org/0000-0002-9234-3646 inmec130@mail.ru \\ Ответственный автор. Электронная почта: inmec130@ mail.ru \\ Адрес для переписки: Мичуринский проспект, 1, Москва, 119192, Российская Федерация
}

Общеизвестно, что элементы конструкций и детали, работающие в нагруженном состоянии при высоких температурах, могут подвергаться процессу необратимого деформирования во времени. Для проведения расчетов эксплуатационного запаса (на основе соответствующих механических моделей) могут потребоваться механические характеристики материала, такие как напряжение при стартовой ползучести и напряжение в момент разрушения для конкретного материала при заданной температуре. Эти механические характеристики не представляется возможным определить из диаграммы механического состояния материала, они измеряются в процессе испытаний на одноосное растяжение цилиндрических образцов при постоянстве растягивающего напряжения во времени. В работе предложено взамен проведения специальных испытаний вычислить искомые механические характеристики из аппроксимаций процессов установившейся ползучести и длительной прочности. С этой целью были рассмотрены две дробно-степенные функции с четырьмя материальными параметрами (С. А. Шестериков и М. А. Юмашева, 1984), два из которых имеют физический смысл напряжений при стартовой ползучести и в момент разрушения: аппроксимация скорости деформации установившейся ползучести от номинального напряжения (1), аппроксимация времени в момент разрушения от номинального напряжения (2). В вычислениях использовали опытные данные, полученные для титанового сплава ВТ6 при температуре $650{ }^{\circ} \mathrm{C}$ на цилиндрических образцах (диаметр - 5 мм, рабочая длина - 25 мм). Параметры дробностепенных аппроксимаций были вычислены итерационным методом Generalized Reduced Gradient Method (Microsoft Excel) при условии минимальной суммарной погрешности расхождения опытных данных относительно аппроксимирующей кривой в соответствующих логарифмических осях. Анализ погрешностей аппроксимаций (1) и (2) показал, что погрешность (1) меньше (2), при этом отношение напряжения в момент разрушения к напряжению при стартовой ползучести больше. Таким образом, при вычислении напряжений при стартовой ползучести и в момент разрушения предпочтительнее использовать аппроксимацию (1).

Ключевые слова: предельные напряжения процесса ползучести, напряжение при стартовой ползучести, напряжение при разрыве.

\section{1. Введение}

Почти все механические характеристики ( $E$ - модуль упругости; $\sigma_{0,2}-$ предел текучести; $\sigma_{\text {strength }}$ - предел прочности) при заданной температуре определяют из анализа экспериментальной диаграммы механического состояния материала. Вместе с этим существуют другие механические характеристики, которые не могут быть определены 
из экспериментальной диаграммы механического состояния материала. Одними из таких механических характеристик являются напряжение при стартовой ползучести $\sigma_{\text {startcreep }}$ - максимальное возможное напряжение, при котором скорости деформаций ползучести равны нулю, а также напряжение кратковременной прочности при разрыве $\sigma_{\text {rupture }}$ - минимальное возможное напряжение, при котором происходит мгновенное разрушение. Эти механические характеристики могут быть использованы для описания процессов установившейся ползучести и длительной прочности. Следует отметить, что в некоторых статьях [1] не различают $\sigma_{\text {strength }}$ и $\sigma_{\text {ruptur }}$ (что не верно). Механические характеристики $E, \sigma_{0,2}$ и $\sigma_{\text {strengt1 }}[1]$ получены из диаграммы механического состояния никелевого сплава ГC32-VI при $570{ }^{\circ} \mathrm{C}$. С учетом этих экспериментальных данных отработана механическая модель [1] разрушения хвостовика лопатки газотурбинной установки. Из анализа [2] механических характеристик ползучести сплава $\mathrm{Mg}-\mathrm{Li}$ при $20{ }^{\circ} \mathrm{C}$ установлено $\sigma_{\text {startcreep }}<\sigma_{0,2}$. В книге [3] изложена модель разрушения толстостенной цилиндрической трубы под внутренним давлением, при этом процесс разрушения условно разделен во времени на две последовательные стадии. Первая стадия характеризуется латентным разрушением с образованием и ростом микропор. Вторая стадия характеризуется продвижением фронта разрушения. Вместо общепринятых степенных аппроксимаций процессов установившейся ползучести и длительной прочности впервые рассмотрены дробно-степенные зависимости с четырьмя параметрами, два из которых принимают физический смысл предельных напряжений процесса ползучести. Из анализа результатов статистической обработки [5] экспериментальных данных установлено, что процесс длительной прочности аппроксимируется дробно-степенной зависимостью [4] лучше, чем общепринятой степенной зависимостью. В статье [6] приведено сравнение нескольких моделей описания процессов длительной прочности в условиях сложного напряженного состояния с учетом процесса разрушения. Эти модели предполагают, что повреждения накапливаются в плоскостях, перпендикулярных действию главных растягивающих напряжения, при этом функция скорости накопления повреждений от главных растягивающих напряжений принимает вид [4]. Результаты вычислений приведены с учетом прочностной анизотропии. Сделан также вывод, что при описании процесса длительной прочности желательно учитывать явление прочностной анизотропии. Для описания чистого изгиба балок в условиях установившейся ползучести рассмотрены балки прямоугольного и круглого сечений [7] с учетом зависимости [4]. В статье [8] рассмотрена модель установившейся ползучести стержня круглого сечения с учетом процессов накопления повреждений и диффузии химических элементов из окружающей среды. В этой модели автор использовал предельные напряжения процесса ползучести, однако как их определить не указал. Одним из способов определения этих предельных напряжений - измерение их в эксперименте. Цилиндрические образцы испытываются при ступенчатом изменении во времени осевой силы до тех пор, пока не будут обнаружены предельные напряжения. В случае если уже известны опытные данные по установившейся ползучести и длительной прочности материала при заданной температуре, можно попытаться вычислить искомые предельные напряжения $\sigma_{\text {startcreep }}$ и $\sigma_{\text {rupture }}$ из двух ниже рассмотренных аппроксимаций. 


\section{2. Две рассматриваемые модели}

Рассмотрим две альтернативные модели [4] вычисления предельных напряжений

$$
\begin{aligned}
& A \dot{u}_{\text {second }}^{\text {approx }}\left(\sigma_{\text {nominal }}\right)=\left[\frac{\sigma_{\text {nominal }}-\sigma_{\text {startcreep }}}{\sigma_{\text {rupture }}-\sigma_{\text {nominal }}}\right]^{n} ; \\
& \sigma_{\text {startcreep }}<\sigma_{\text {nominal }}<\sigma_{\text {rupture }} A>0 \quad n>0 \\
& \frac{t_{\text {rupture }}^{\text {approx }}\left(\sigma_{\text {nominal }}\right)}{B}=\left[\frac{\sigma_{\text {rupture }}-\sigma_{\text {nominal }}}{\sigma_{\text {nominal }}-\sigma_{\text {startcreep }}}\right]^{m}, \\
& \sigma_{\text {startcreep }}<\sigma_{\text {nominal }}<\sigma_{\text {rupture }} B>0 \quad m>0
\end{aligned}
$$

где $\sigma_{\text {nominal }}-$ номинальное напряжение (нормальное растягивающее напряжение в момент нулевого удлинения); $\dot{u}_{\text {second }}^{\text {approx }}$ - скорость удлинения при установившейся ползучести из аппроксимации (1); $t_{\text {rupt }}^{\text {approx }}$ - время в момент разрушения из аппроксимации (2); $A, B, n, m-$ материальные параметры при заданной высокой температуре (в данной статье рассматривается изотермическая ползучесть), а также два размерных параметра $[A]=$ ч/мм и $[B]=ч$. Зависимости (1) и (2) описывают соответствующие механические процессы (рис. 1-2).

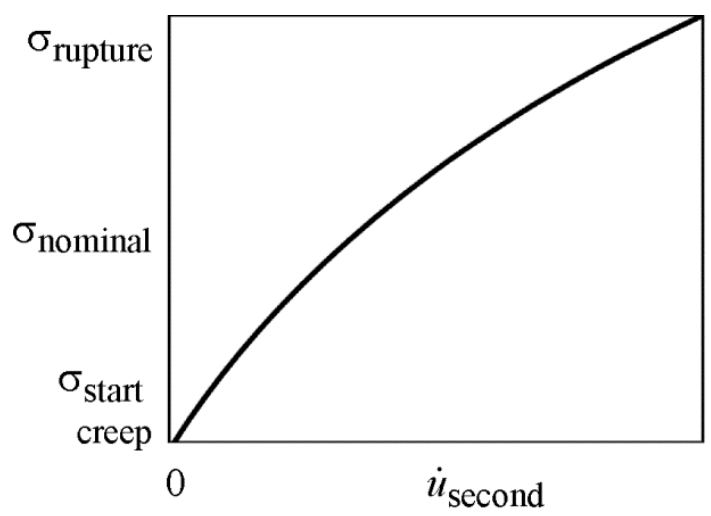

Рис. 1. Зависимость напряжения от скорости удлинения при установившейся ползучести $\sigma_{\text {nominal }}\left(\dot{u}_{\text {second }}\right)$

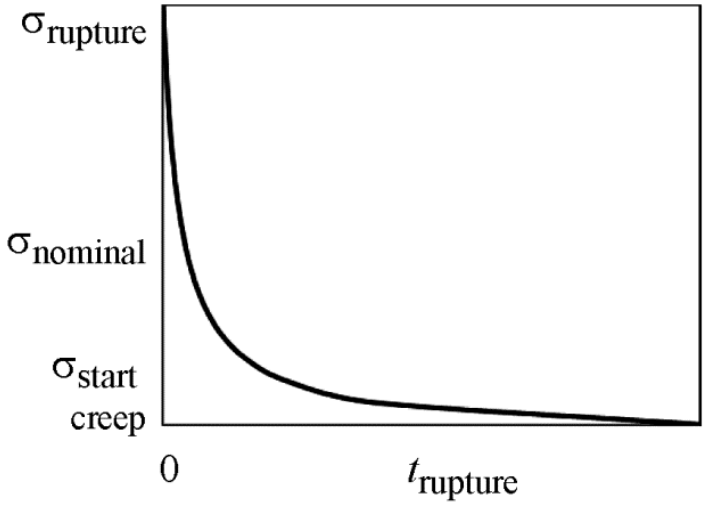

Рис. 2. Зависимость напряжения от времени в момент разрушения

$$
\sigma_{\text {nominal }}\left(t_{\text {rupture }}\right)
$$

Следует обратить внимание, что зависимости (1) и (2) ограничены напряжениями $\sigma_{\text {startcreep }}$ и $\sigma_{\text {rupture }}$ Между тем, степенные зависимости с двумя параметрами не имеют этих ограничений. Зависимости (1) и (2) предложены [4] с учетом того, что предельные напряжения $\sigma_{\text {startcreep }}$ и $\sigma_{\text {rupture }}$ уже измерены в эксперименте и могут быть использованы в этих зависимостях. Остальные параметры подлежат вычислению одним из итерационных методов при условии минимальной суммарной погрешности расхождения опытных данных и аппроксимирующих значений. Новизна данной работы заключается в попытке вычислить все четыре параметра одновременно. 


\section{3. Способ сравнения двух моделей}

Чтобы выяснить преимущество одной из двух моделей, следует вычислить погрешности суммарного расхождения опытных данных и их аппроксимирующих значений

$$
\begin{aligned}
& \Delta_{1}=\min \left(\sum_{1}^{N}\left|\lg \frac{\dot{u}_{\text {second }}^{\text {approx }}}{\dot{u}_{\text {second }}^{\text {exper }}}\right|\right) ; \\
& \Delta_{2}=\min \left(\sum_{1}^{N}\left|\lg \frac{t_{\text {rupture }}^{\text {approx }}}{t_{\text {rupture }}^{\text {exper }}}\right|\right),
\end{aligned}
$$

где $N$ - количество опытных точек; $\dot{u}_{\text {second }}^{\text {exper }} t_{\text {rupturs }}^{\text {exper }}-$ скорость удлинения при установившейся ползучести и время в момент разрушения из эксперимента. Выражения (3) и (4) определяют сумму расстояний между соответствующими точками в логарифмических осях. Так как в логарифмических осях расстояние между точками меньше, чем в обычных осях координат, это позволяет более точно вычислить неизвестные параметры (Generalized Reduced Gradient Method inside Microsoft Excel). Сравнительный анализ значений погрешностей (3) и (4) позволит установить превосходство одной из двух моделей.

\section{4. Анализ результатов}

Результаты вычислений получены с учетом опытных данных [9]. Цилиндрические образцы (рис. 3) из титанового сплава BT6 (Grade-5) подвергались одноосному растяжению в условиях постоянства осевой силы.

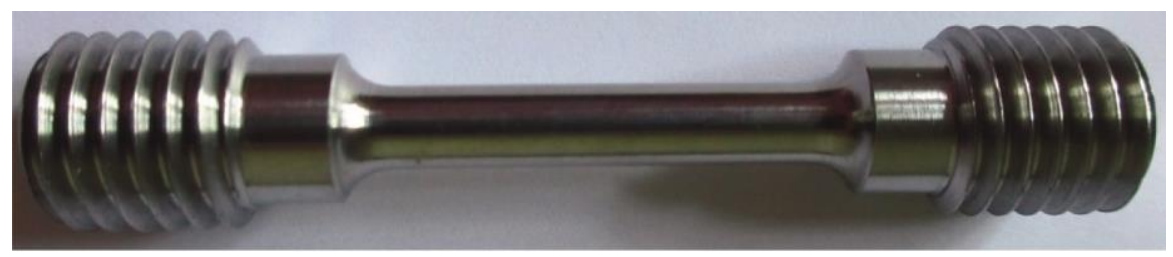

Рис. 3. Цилиндрический образец (диаметр 5 мм и базовая длина 25 мм) для механических испытаний при одноосном растяжении

Эти механические испытания проводились на самодельной установке [10]. Нагрев образцов производился внутри цилиндрической печи до температуры $650{ }^{\circ} \mathrm{C}$. Контроль над температурой во времени осуществлялся специальным устройством «Протерм-100» вместе с термопарами по трем контрольным точкам. Измерительная система позволяла измерить текущее удлинение и время в момент разрушения. Параметры модели (1) вычислены с погрешностью $\Delta_{1}=1,00$ и принимают значения $\sigma_{\text {startcreep }}=13 \mathrm{MПа;} \sigma_{\text {rupture }}=273 \mathrm{MПа;} A=0,15$ ч/мм; $n=1,1$. Параметры модели (2) вычислены с погрешностью $\Delta_{2}=1,36$ и принимают значения $-\sigma_{\text {startcreep }}=21$ МПа; $\sigma_{\text {rupture }}=21 \mathrm{MПа;} B=2,70$ ч; $m=1,1$. Аппроксимации $\sigma_{\text {nominal }}\left(A \dot{u}_{\text {second }}^{\text {approx }}\right)$ и $\sigma_{\text {nominal }}\left(t_{\text {rupture }}^{\text {approx }} / B\right)$ принимают различный характер монотонности (рис. 4), где $A \dot{u}_{\text {second }}^{\text {approx }} t_{\text {rupture }}^{\text {approx }} / B$ - безразмерная скорость удлинения при установившейся ползучести и безразмерное время в момент разрушения (табл.). 

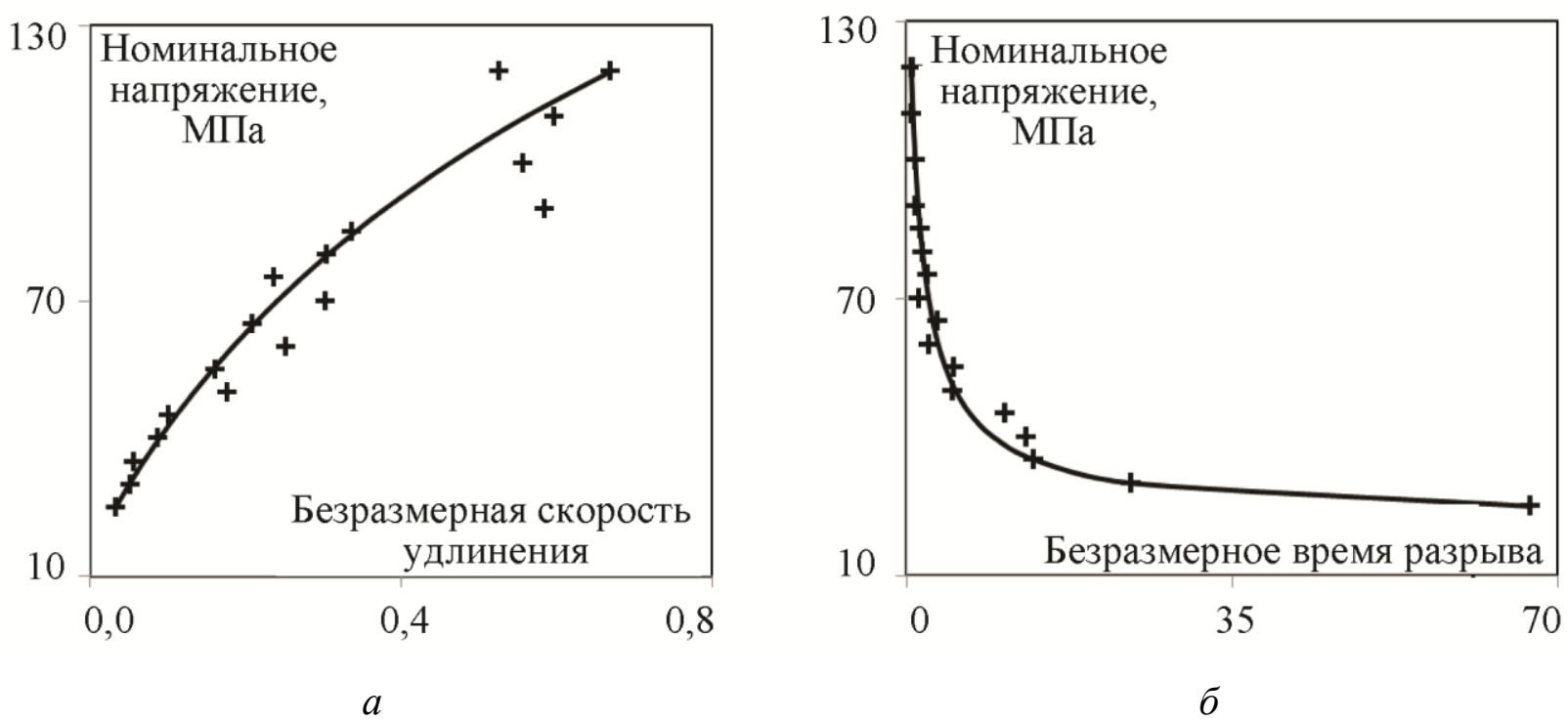

Рис. 4. Зависимости номинального напряжения от безразмерной скорости удлинения $(a)$ : 1 - экспериментальные данные $\sigma_{\text {nominal }}\left(A \dot{u}_{\text {second }}^{\text {exper }}\right) ; 2$ - аппроксимация $\sigma_{\text {nominal }}\left(A \dot{u}_{\text {second }}^{\text {approx }}\right)$; от безразмерного времени разрыва (б): 1 - экспериментальные данные $\sigma_{\text {nominal }}\left(t_{\text {rupture }}^{\text {exper }} / B\right)$;

$$
2 \text { - аппроксимация } \sigma_{\text {nominal }}\left(t_{\text {rupture }}^{\text {approx }} / B\right)
$$

Диаграмма механического состояния титанового сплава ВТ6 при $650{ }^{\circ} \mathrm{C}$ не приводится в статье [9], что не позволяет провести сравнение вычисленных напряжений $\sigma_{\text {startcreep }}$ и $\sigma_{\text {rupturf }}$ с характерными точками $\sigma_{0,2}$ и $\sigma_{\text {strengtl. }}$ Отношение погрешностей $\Delta_{2} / \Delta_{1}=1,36$. Различие этих погрешностей обусловлено лучшей стабильностью экспериментальных данных по установившейся ползучести чем по длительной прочности. Это означает, что несколько экспериментов при одних и тех же условиях приводят к мало отличающимся экспериментальным данным. Обе модели (1) и (2) могут быть использованы для расчета предельных напряжений ползучести. Однако для расчета предельных напряжений процесса ползучести модель (1) предпочтительнее модели (2).

В подтверждение этого имеются следующие два аргумента. Первый из них - хорошая стабильность экспериментальных данных $\dot{u}_{\text {second }}^{\text {exper }}\left(\sigma_{\text {nominal }}\right)$ позволяет провести более точные вычисления (экспериментальные данные $t_{\text {rupture }}^{\text {exper }}\left(\sigma_{\text {nominal }}\right)-$ менее стабильны). Второй измерение зависимости $\dot{u}_{\text {second }}^{\text {exper }}\left(\sigma_{\text {nominal }}\right)$ из эксперимента менее трудоемкий процесс, чем зависимости $t_{\text {rupture }}^{\text {exper }}\left(\sigma_{\text {nominal }}\right)$. 
Таблица 1 - Данные для рис. 4.

\begin{tabular}{|c|c|c|c|c|}
\hline \multicolumn{5}{|c|}{ Pис. 4 } \\
\hline \begin{tabular}{c}
\hline \multicolumn{5}{|c|}{$a$} \\
$\sigma_{\text {nominal }}$
\end{tabular} & $A \dot{u}_{\text {second }}^{\text {exper }}$ & $A \dot{u}_{\text {second }}^{\text {approx }}$ & $\frac{t_{\text {rupture }}^{\text {exper }}}{B}$ & $\frac{t_{\text {rupprox }}^{\text {approx }}}{B}$ \\
\hline 25 & 0,03 & 0,04 & 67,2 & 53,9 \\
\hline 30 & 0,05 & 0,05 & 24,2 & 21,3 \\
\hline 35 & 0,06 & 0,07 & 13,8 & 12,6 \\
\hline 40 & 0,09 & 0,09 & 13,0 & 8,6 \\
\hline 45 & 0,10 & 0,12 & 10,6 & 6,4 \\
\hline 50 & 0,17 & 0,14 & 5,0 & 5,0 \\
\hline 55 & 0,16 & 0,16 & 5,2 & 4,0 \\
\hline 60 & 0,25 & 0,19 & 2,5 & 3,3 \\
\hline 65 & 0,21 & 0,22 & 3,3 & 2,7 \\
\hline 70 & 0,30 & 0,25 & 1,4 & 2,3 \\
\hline 75 & 0,23 & 0,28 & 2,4 & 2,0 \\
\hline 80 & 0,30 & 0,31 & 1,8 & 1,7 \\
\hline 85 & 0,33 & 0,35 & 1,5 & 1,5 \\
\hline 90 & 0,58 & 0,39 & 0,9 & 1,3 \\
\hline 100 & 0,55 & 0,47 & 0,9 & 0,9 \\
\hline 110 & 0,59 & 0,56 & 0,6 & 0,7 \\
\hline 120 & 0,52 & 0,67 & 0,8 & 0,5 \\
\hline 120 & 0,66 & 0,67 & 0,5 & 0,5 \\
\hline
\end{tabular}

Вычисленные предельные напряжения процесса ползучести $\sigma_{\text {startcreep }}$ и $\sigma_{\text {rupture }}$ из аппроксимаций (1) and (2) располагаются вне диапазона $25 \mathrm{MPa} \leq \sigma_{\text {nominal }} \leq 120 \mathrm{MPa}$ экспериментальных данных [9], что не противоречит неравенству $\sigma_{\text {startcreep }}<\sigma_{\text {nominal }}<\sigma_{\text {rupture }}$. Достоверность предложенной методики основана на свойстве монотонности функций (1) и (2). Различие между предельными напряжениями, вычисленных с учетом аппроксимаций (1) и (2), объясняется лишь различной устойчивостью экспериментальных данных.

\section{5. Выводы}

Идея о том, что предельные напряжения процесса ползучести можно не только измерить в эксперименте, а также вычислить из аппроксимации опытных данных, позволила расширить возможности применения моделей (1) и (2).

Из анализа вычисленных погрешностей следует, что для вычисления предельных напряжений процесса ползучести следует использовать модель (1).

\section{Литература}

1. Наприенко С. А., Орлов М. Р. Разрушение монокристаллических лопаток турбины наземных ГТУ // Труды ВИАМ. - 2016. - Т. 38, № 2. - C. 20-31. - DOI: 10.18577/2307-60462016-0-2-3-3.

2. Ильин С. И., Корягин Ю. Д., Лапина Е. В. Ползучесть ультралегких магниевых сплавов при низких температурах // Вестник ЮУрГУ. - 2012. - Вып. 18, № 15. - С. 105-107. URL: https://vestnik.susu.ru/metallurgy/article/viewFile/1371/1335

3. Качанов Л. М. Основы механики разрушения. - М. : Наука, 1974. - 312 с. 
4. Шестериков С. А., Юмашева М. А. Конкретизация уравнений состояния в теории ползучести // Изв. АН СССР. Механика твердого тела. - 1984. - № 1. - С. 86-92.

5. Nazarov V. V., Lepeshkin A. R. A method of calculating creep limits // Diagnostics, Resource and Mechanics of materials and structures. - 2017. - Iss. 1. - P. 36-42. - DOI: 10.17804/2410-9908.2017.1.036042. - URL: http://dream-journal.org/DREAM_Issue_1_2017_Nazarov_V.V._et_al._036_042.pdf

6. Локощенко А. М., Назаров В. В. Кинетический подход исследования длительной прочности металлов при двухосном растяжении // Авиационно-космическая техника и технология. - 2005. - Вып. 26, № 10. - С. 73-79.

7. Локощенко А. М., Агахи К. А., Фомин Л. В. Чистый изгиб балки в условиях ползучести из разно-сопротивляющегося материала // Вестник Самарского государственного технического университета. Серия: Физико-математические науки. - 2012. - Вып. 26, № 1. C. 66-73. - DOI: https://doi.org/10.14498/vsgtu1042.

8. Фомин Л. В. Описание длительной прочности растягиваемых стержней прямоугольного и круглого поперечных сечений в высокотемпературной воздушной среде // Вестник Самарского государственного технического университета. Серия: Физико-математические науки. - 2013. - Вып. 32, № 3. - C. 87-97. - DOI: https://doi.org/10.14498/vsgtu1228.

9. Назаров В. В. Кратковременная ползучесть титановых сплавов ВТ5 и ВТ6 при высокой температуре // Заводская лаборатория. Диагностика материалов. - 2015. - Т. 81, № 6. C. $57-60$.

10. Ковальков В. К., Назаров В. В., Новотный С. В. Методика проведения высокотемпературных испытаний при сложном напряженном состоянии // Заводская лаборатория. Диагностика материалов. - 2006. - Т. 72, № 4. - С. 42-44. 